\title{
Microbiology needs champions on-screen
}

\author{
By sparking imagination and interest during childhood, and by finding and supporting champions to nurture \\ microbial fascination through to adulthood, the fundamental importance of microorganisms can attain a justified \\ and necessary place in the public psyche, but more on-screen time will be needed.
}

\begin{abstract}
nterest in microbiology is resurgent over the past decade, as the fundamental importance of microorganisms to every aspect of life on earth has become ever clearer. Yet, with a few notable exceptions (antimicrobial resistance, for example), the renewed microbial fascination is held chiefly among scientists and science enthusiasts; bandwidth for science stories among the broader public is seemingly limited, and large swathes of the life and physical sciences, microbiology included, can struggle to penetrate the public consciousness unless apocalyptic warnings are invoked. A case in point are the multitude of 'Best of 2017' lists recently published, which were invariably dominated by stories from a relative few scientific disciplines, including space and astronomy, the environment, and ancient evolution. All are serious and awe-inspiring topics no doubt, but such a narrow selection cannot truly encompass the greatest or most important of scientific advances
\end{abstract} unveiled during the past 12 months. Whether this is an example of selective bias in representing science by news and social media organizations (and the press office machineries that underpin them), or simply reflective of audience demand, is open for debate. Certainly esoteric and abstract ideas describing difficult-to-comprehend scales in space or time can spark greater wonder among the general populace than can picking apart the mechanistic details in understanding how two cells interact. Where the subject matter of a story raises a potential threat to the audience, or potential panacea for their problems, no matter how probable either case may be, attention can also be piqued. This is where microbiology garners some coverage, for instance on drugresistant infections, Zika or the microbiome, and it plays into a black-and-white narrative of 'good' (the microbiome) and 'bad' (the pathogens). Yet tales only of threat or cure do not do justice to our field, and do not garner anything like the enduring allure of distant stars, ancient reptiles or ocean depths.

Getting them while they're young. Some areas of science and nature are simply more marketable than others, and the indoctrination starts in childhood, where space rockets and animals (both extinct and extant) feature heavily among the toys in the box, books on the shelf and cartoons on the screen. Here, microbiology is beginning to make some inroads. Plush microbe toys are not solely gifted to microbiologists on special occasions, but are also available to the rest of the public in outlets worldwide, while the number of educational and amusing children's books dedicated to microbiology is growing steadily. Both remain but a drop in the ocean by cosmic or Mesozoic standards, but they are a start upon which a lingering love of all things microbial could be kindled.

Keeping them hooked. Consolidating this early exposure for much of the public, extensive coverage of certain terrestrial and extra-terrestrial arenas on-screen ensures that they have continued reach into adult life. Documentary series, often fronted by celebrated scientists and naturalists, reinforce childhood enchantment and keep viewers entertained while informing them about the world around them and workings of the wider universe beyond. Renowned figures, such as Sir David Attenborough, through his documentaries with the BBC Natural History Unit, or the late Carl Sagan, who co-wrote and narrated the famed 1980s television series Cosmos (and accompanying book), provide voices for science that are welcomed into homes around the world and are trusted for the expertise that they bring and for their ability to communicate complex ideas in a simplified and understandable manner. Not only providing an entertaining snapshot into a particular environment or concept, such documentaries also help to lay the basis for messages about pressing scientific issues (such as the anthropogenic drivers of climate change) to be communicated to inexpert audiences, and therefore have an important role in addressing the immense challenges facing humankind over the next century.

So do we need 'celebrity' microbiologists? It is clear that a better job could be done in communicating the beauty, elegance, importance and deep mystery of the microbial world to the public at large, and in doing so provide necessary context for some of the urgent messages from our field, such as those relating to antibiotic stewardship or vaccine uptake. Microbiologists in the public eye could help to combat the generally germ-centric view of the microbial world and create a better appreciation for the diverse nature and functional importance of microorganisms of all shapes and sizes. Who then, might fulfil this role? The field already has champions, in researchers, writers and science communicators, yet their impact only rarely reaches beyond the scientist and science enthusiast bubble. Whether we aim to raise the profile of these individuals even further, or begin to search for others, no matter whether they are senior or junior, laboratory scientist or away from the bench, the most important qualifications for the role are passion for microbiological science and an ability to engage and hold a broad audience.

Yet even Sagan and Attenborough did not build a public following alone. There is a part to be played by all microbiologists in promoting the field and recognizing and supporting colleagues who step into the limelight to talk about our science, rather than knocking them as attention seeking or for not focusing purely on research (as often happens). As broad public interest is something of a closed shop, and because the medium by which the message is delivered is perhaps even more important that having 'celebrity' scientists delivering the message, a concerted effort will be needed to convince commissioning editors to bring microbiology to greater prominence on-screen. The written word is powerful and there are plenty of skilful exponents with an interest in bringing the world of microorganisms to life.

Yet whether through documentary features or increasingly through content created specifically for social media, film and video will be essential to grabbing and sustaining greater public attention for microorganisms. We've had Cosmos, Planet Earth and Blue Planet; it is time for the Microbial Universe to become destination viewing.

Published online: 22 January 2018 https://doi.org/10.1038/s41564-018-0112-z 